\title{
Investigation of the structure of alpha-lactalbumin protein nanotubes using optical spectroscopy
}

\author{
Özgür Tarhan ${ }^{1 *}$, Enver Tarhan ${ }^{2}$ and Şebnem Harsa ${ }^{1}$ \\ ${ }^{1}$ Department of Food Engineering, Faculty of Engineering, Izmir Institute of Technology, Urla, Izmir TR-35430, Turkey \\ ${ }^{2}$ Department of Physics, Faculty of Science, Izmir Institute of Technology, Urla, Izmir TR-35430, Turkey
}

Received 22 February 2013; accepted for publication 31 October 2013

\begin{abstract}
Alpha-lactalbumin $(\alpha-\mathrm{la})$ is one of the major proteins in whey. When partially hydrolysed with Bacillus licheniformis protease, it produces nanotubular structures in the presence of calcium ions by a self-assembly process. This study presents investigation of $\alpha$-la protein structure during hydrolysis and nanotube formation using optical spectroscopy. Before spectroscopic measurements, nanotubes were examined with microscopy. The observed $\alpha$-la nanotubes ( $\alpha$-LaNTs) were in the form of regular hollow strands with a diameter of about $20 \mathrm{~nm}$ and the average length of $1 \mu \mathrm{m}$. Amide and backbone vibration bands of the Raman spectra displayed remarkable conformational changes in $\alpha$ and $\beta$ domains in the protein structure during nanotube growth. This was confirmed by the Fouriertransform infrared (FTIR) spectroscopy data. Also, FTIR analysis revealed certain bands at calcium $\left(\mathrm{Ca}^{++}\right)$binding sites of $\mathrm{COO}^{-}$groups in hydrolysed protein. These sites might be critical in nanotube elongation.
\end{abstract}

Keywords: $\alpha$-Lactalbumin, protein nanotubes, microscopy, Raman, FTIR.

Food proteins and peptides have a special importance due to their ability to form supramolecular structures naturally by a process of self-assembly. This process can be defined by the spontaneous and reversible aggregation due to polymerisation of molecules by non-covalent intermolecular interactions such as hydrogen bonding, electrostatic, hydrophobic, and Van der Waals interactions (Rajagapol \& Schneider, 2004). Enzymatic hydrolysis leads to breakdown of the proteins through smaller protein/peptide fragments depending on degree of hydrolysis. Naturally occurring selfassembly may give rise to fabrication of highly ordered novel nanoscale structures from these fragments. $\alpha$-Lactalbumin $(\alpha-\mathrm{la})$ is a globular and compact protein with a molecular weight of $14 \cdot 2 \mathrm{kDa}$ with an ability to form fibrillar nanostructures called 'nanotubes'. Ipsen et al. (2001) reported the first partially hydrolysed $\alpha$-la with Bacillus licheniformis protease, BLP, which formed strong gels consisting nonbranching, hollow, and novel microtubules with a uniform diameter distribution of about $20 \mathrm{~nm}$ and lengths exceeding $2 \mu \mathrm{m}$. Graveland-Bikker et al. (2004) investigated the effect of calcium on nanotube growth and gel formation due to self-assembly after limited hydrolysis of $\alpha$-la. They reported transparent gels with fine strands (nanotubes) having outer

*For correspondence; e-mail: ozgurtarhan@iyte.edu.tr and inner diameters around 20 and $7 \mathrm{~nm}$, respectively. Protein hydrolysis, hydrolysis products (building blocks of nanotubes), and nanotube growth kinetics were investigated in detail (Graveland-Bikker et al. 2006, 2009). Nanotube triggered gel formation affected by the certain parameters were also examined (Ipsen \& Otte, 2003; Ipsen et al. 2003). Although there are convincing microscopic and kinetic studies, there is a lack of detailed investigation on the structural characterisation of nanotube elongation. Ipsen \& Otte (2007) proposed a three stage mechanism for the formation of $\alpha$-la nanotubes ( $\alpha$-LaNTs): destabilising of the native protein structure, nucleus formation by dimeric building blocks, and elongation of the nuclei into nanotubes. GravelandBikker et al. (2009) investigated building blocks of the nanotube structure and proposed a model for the self-assembly of $\alpha$-la into nanotubes. However, the structural changes in the protein during hydrolysis and nanotube growth have not yet been revealed clearly in literature. The main goal of this research was to investigate such conformational changes occurring in the protein structure during nanotube formation.

Raman spectroscopy is an effective tool to investigate the conformational transitions in proteins by detecting changes in the secondary structure. It provides information related to the vibrational motions of the molecules to be used in prediction or identification of their chemical structures (Nonaka et al. 1993). Backbone vibrations in polypeptides and 
proteins are usually associated with three main regions in the Raman spectrum (Tu, 1986; Miura \& Thomas, 1995; Blanch et al. 2000). These are the backbone skeletal stretch region of $\sim 870-1150 \mathrm{~cm}^{-1}$, arising from $\mathrm{C}_{\alpha}-\mathrm{C}, \mathrm{C}_{\alpha}-\mathrm{C}_{\beta}$, and $\mathrm{C}_{\alpha}-\mathrm{N}$ stretches; the amide III region of $\sim 1230-1340 \mathrm{~cm}^{-1}$, resulting mainly from in-phase combination of the $\mathrm{N}-\mathrm{H}$ inplane deformation vibrations with the $\mathrm{C}_{\alpha}-\mathrm{N}$ stretch; and amide I region of $\sim 1630-1700 \mathrm{~cm}^{-1}$, originating mainly from the $\mathrm{C}=\mathrm{O}$ stretch.

BLP cuts $\alpha$-la at aspartic (Asp) and glutamic (Glu) acid sites (Breddam \& Meldal, 1992) during hydrolysis prior to nanotube development. Carboxylic acid $\left(\mathrm{COO}^{-}\right)$groups exposed at these ends become available for cation chelation. $\mathrm{Ca}^{++}$ ions act as intermolecular salt bridges between carboxylates of different peptide units and they are involved in the nanotube elongation (Ipsen \& Otte, 2007). Fourier-transform infrared (FTIR) spectroscopy is used to determine the interactions of $\mathrm{Ca}^{++}$binding to $\alpha$-la through the coordination of $\mathrm{COO}^{-}$groups (Nara et al. 1994; Mizuguchi et al. 1997). Absorption in amino acid side chains provides valuable information about molecular reaction mechanisms and structural changes in proteins. Amide modes are used to analyse polypeptide backbone vibrations in proteins (Krimm \& Bandekar, 1986; Barth, 2007). Amide I band around $1650 \mathrm{~cm}^{-1}$, arises from $\mathrm{C}=\mathrm{O}$ stretching vibrations; amide II band around $1550 \mathrm{~cm}^{-1}$ arises from the combination of $\mathrm{N}-\mathrm{H}$ bending and $\mathrm{C}-\mathrm{N}$ stretching vibrations; and amide III mode in the region of $1400-1200 \mathrm{~cm}^{-1}$ arises from the combination of $\mathrm{N}-\mathrm{H}$ bending and $\mathrm{C}-\mathrm{N}$ stretching vibrations. The amide I vibration region is mostly used to analyse the secondary structure in proteins. This vibration is hardly affected by the nature of the side chain depending on the secondary structure of the backbone. Amide II and III bands also provide valuable structural information in proteins.

Due to a number of characteristics, $\alpha$-LaNTs have been suggested for various potential food (viscosifying, gelation and encapsulation) and non-food (scaffolding) applications (Graveland-Bikker \& de Kruif, 2006). For such applications, both mechanical and structural properties of nanotubes should be known clearly. Especially, the knowledge of the secondary structure of the nanotubes and nanotube formation process is very important for a better understanding of the characteristics of the nanotubes.

The objectives of this study were to investigate structural conformations in the $\alpha$-la during the hydrolysis and nanotube formation, and the $\mathrm{Ca}^{++}$binding sites involved in nanotube elongation using FTIR and Raman Spectroscopy.

\section{Materials and methods}

\section{Materials}

All chemicals and standards used in this work were purchased from Sigma (Germany). Bacillus licheniformis protease (BLP), 2.4 AU-A/g, was kindly provided by Novozymes A/S (Bagsvaerd, Denmark). Low protein binding filters $(0 \cdot 1 \mu \mathrm{m})$ were purchased from Millipore (Merck KGaA,
Darmstadt, Germany). Copper grids were supplied by Electron Microscopy Sciences (USA).

\section{Enzymatic Hydrolysis of $\alpha$-la (sample preparation)}

The formation of $\alpha$-LaNTs was achieved according to Graveland-Bikker et al. (2004). Three per cent (w/v) of $\alpha$-la (Sigma L6010) was dissolved in 75 mм Tris- $\mathrm{HCl}, \mathrm{pH} 7 \cdot 5$, and $\mathrm{CaCl}_{2}$ with the ratio of $2: 1\left(\mathrm{~mol} \mathrm{Ca}{ }^{++}:\right.$mol $\left.\alpha-\mathrm{la}\right)$, and $4 \% \mathrm{BLP}$ $(\mathrm{w} / \mathrm{w})$ were added. After mixing, the solution was filtered immediately through $0 \cdot 1 \mu \mathrm{m}$ low protein binding filter to remove impurities. Then, the solution was incubated at $50{ }^{\circ} \mathrm{C}$ for $1.5 \mathrm{~h}$ for enzymatic hydrolysis and nanotube development by self-assembly. At the end of the incubation, samples were stored at $+4{ }^{\circ} \mathrm{C}$ for further analysis. For Raman and FTIR analyses, native $\alpha$-la was prepared by dissolving in tris buffer, and $\alpha$-la hydrolysates were prepared by limited proteolysis of $\alpha$-la with BLP.

\section{Microscopy}

To confirm nanotube growth before optical investigations, constructed $\alpha$-LaNTs were examined by Transmission Electron Microscopy (TEM) and Atomic Force Microscopy (AFM). Samples for TEM were taken from the reaction mixture at the end of the incubation and diluted as 1000 fold. Ten $\mu$ l of sample was placed onto carbon coated copper grids and dried at RT. The structures were then examined by TEM at 80 kV (FEI Technai Spirit, The Netherlands). Samples were also examined by Scanning Electron Microscopy (SEM) with an STEM detector operating at $12 \mathrm{kV}$ (FEI Quanta 250FEG, The Netherlands).

For AFM, $10 \mu \mathrm{l}$ of a sample (1000 fold-diluted) was placed onto mica and/or glass surfaces. After drying for overnight at RT, the structures were examined by AFM, MMSPM Nanoscope IV (Veeco, USA). A silicon cantilever, $f_{0}$ : 330-359 kHz and k: 12-103 N/m, (Veeco, USA) was used.

\section{Raman spectroscopy}

A confocal Raman system (S\&I Germany, based on Princeton Instruments, USA) was used to obtain the Raman data of $\alpha$-la samples. Ten $\mu$ l samples were dropped onto glass slides to be placed under the objective of an Olympus microscope which directs the selected laser beam $(488 \mathrm{~nm}$ argon ion laser, $120 \mathrm{~mW}$ power) onto the sample and collects the Raman signal. The signal is then sent to a monochromator (700 mm focal length, Princeton Instrument) to obtain the spectrum data which is recorded by a CCD camera. The data is then sent to the computer which controls the Raman system as well. Typical resolutions based on the chosen gratings in the monochromator $(150,600$, or 1800 groves $/ \mathrm{mm}$ ) change from 1 to $10 \mathrm{~cm}^{-1}$ and the entrance slit width (mostly $100 \mu \mathrm{m}$ is used). Typical Raman data is obtained as the average of 50 scans with one second accumulation each. Raman shifts were recorded in the 

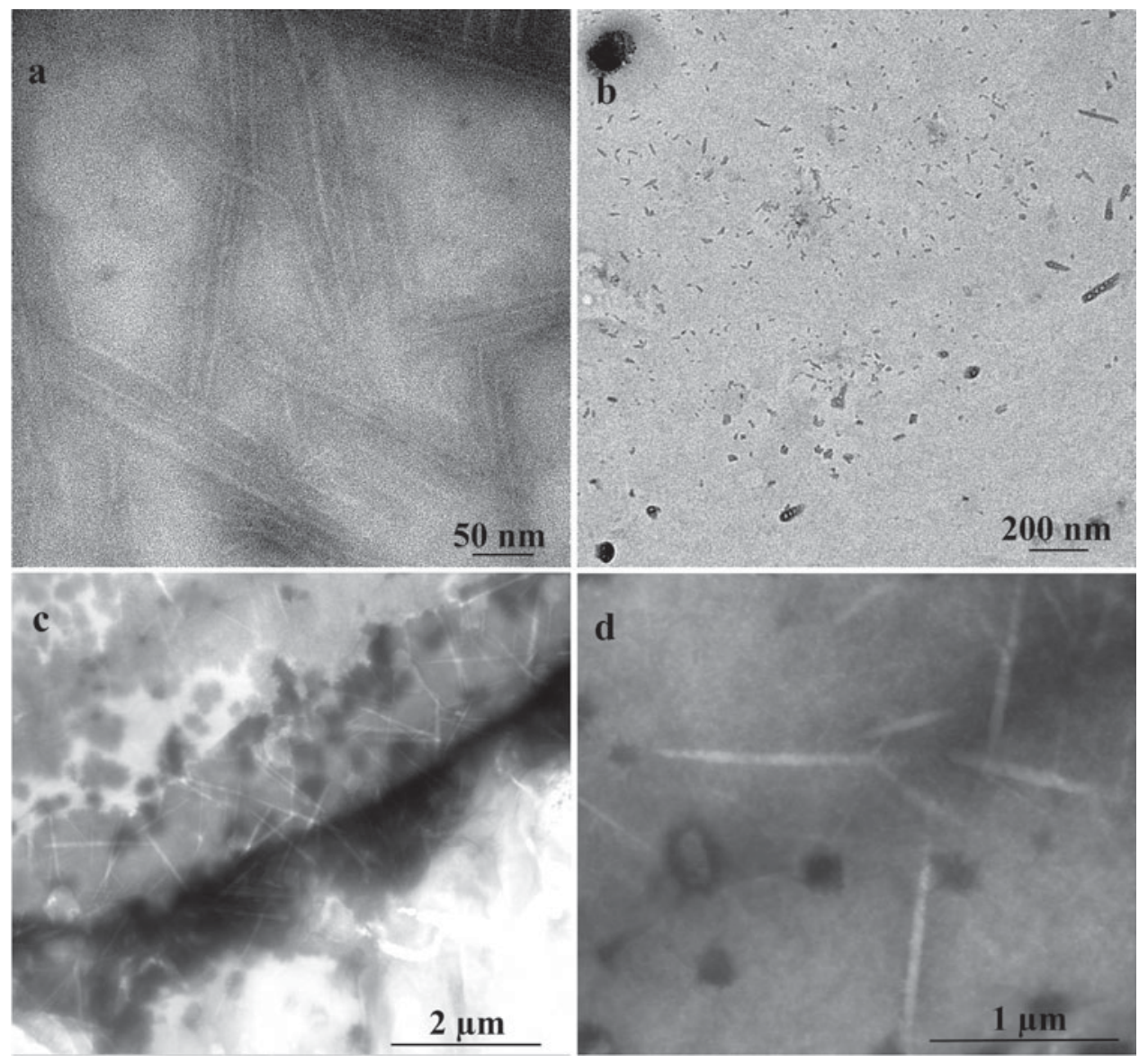

Fig. 1. Transmission electron micrographs of (a) $\alpha$-LaNTs and (b) $\alpha$-La hydrolysed by BLP. (c) and (d) Scanning transmission electron micrographs of $\alpha$-LaNTs.

Stokes side usually from 100 to $4000 \mathrm{~cm}^{-1}$ away from the Rayleigh line.

\section{FTIR spectroscopy}

FTIR measurements were carried out at RT using a PerkinElmer Spectrum 100 FTIR Spectrometer (Perkin Elmer Inc., Wellesley, MA) equipped with a horizontal ATR sampling surface (ZnSe crystal) and a deuterated tri-glycine sulphate (DTGS) detector. One hundred $\mu \mathrm{l}$ samples were placed onto a crystal surface and the measurements were taken in the range of $4000-600 \mathrm{~cm}^{-1}$. The resolution was $4 \mathrm{~cm}^{-1}$ and 64 scan were recorded per each spectrum with a scan speed of $1 \mathrm{~cm} / \mathrm{s}$. A background was recorded before each measurement. Deconvolution of the data was performed with the parameters, $\gamma: 0.75$ and $L: 15 \mathrm{~cm}$.

\section{Results and discussion}

\section{Microscopic analysis of $\alpha$-LaNTs}

Partial hydrolysis of $\alpha$-la by BLP in the presence of calcium resulted in the formation of nanotubular structures through self-assembly. Figure 1a-d shows TEM and STEM images of
$\alpha$-LaNTs, respectively. The observed structures were longer than $100 \mathrm{~nm}$ and about $20 \mathrm{~nm}$ in diameter. The dimensions and morphologies of the structures showed consistency with the literature (Ipsen et al. 2001; Graveland-Bikker et al. 2004). $\mathrm{Ca}^{++}$ions added to medium act as bridges between peptides produced during the hydrolysis and trigger nanotube formation (Ipsen \& Otte 2007). In order to test this, only $\alpha$-la and BLP were mixed and incubated. Figure $1 b$ indicated that, no nanotubular structures were obtained without addition of calcium. This finding supports the crucial importance of calcium when producing $\alpha$-la nanotubes by selfassembly process.

$\alpha$-LaNTs were reported as hollow tubes in the literature (Graveland-Bikker \& de Kruif, 2006). In Fig. 1c and d STEM images show such hollow tubules with brighter inner parts. Also, interferences occurred at the points where the tubules crossover (Fig. 1c). Some nanotubes seemed as wider or thicker which is supposed to be due to the attachment and/or overlapping of the strands. Typically, nanotubes were unbranched but, occasionally, three-way junctions were also observed (Fig. 1d). Cryo-electron microscope image of such a junction has already been (Graveland-Bikker et al. 2009).

AFM images of $\alpha$-LaNTs were also obtained. Figure 2a and $b$ show typical images of $\alpha$-LaNTs prepared by molar 

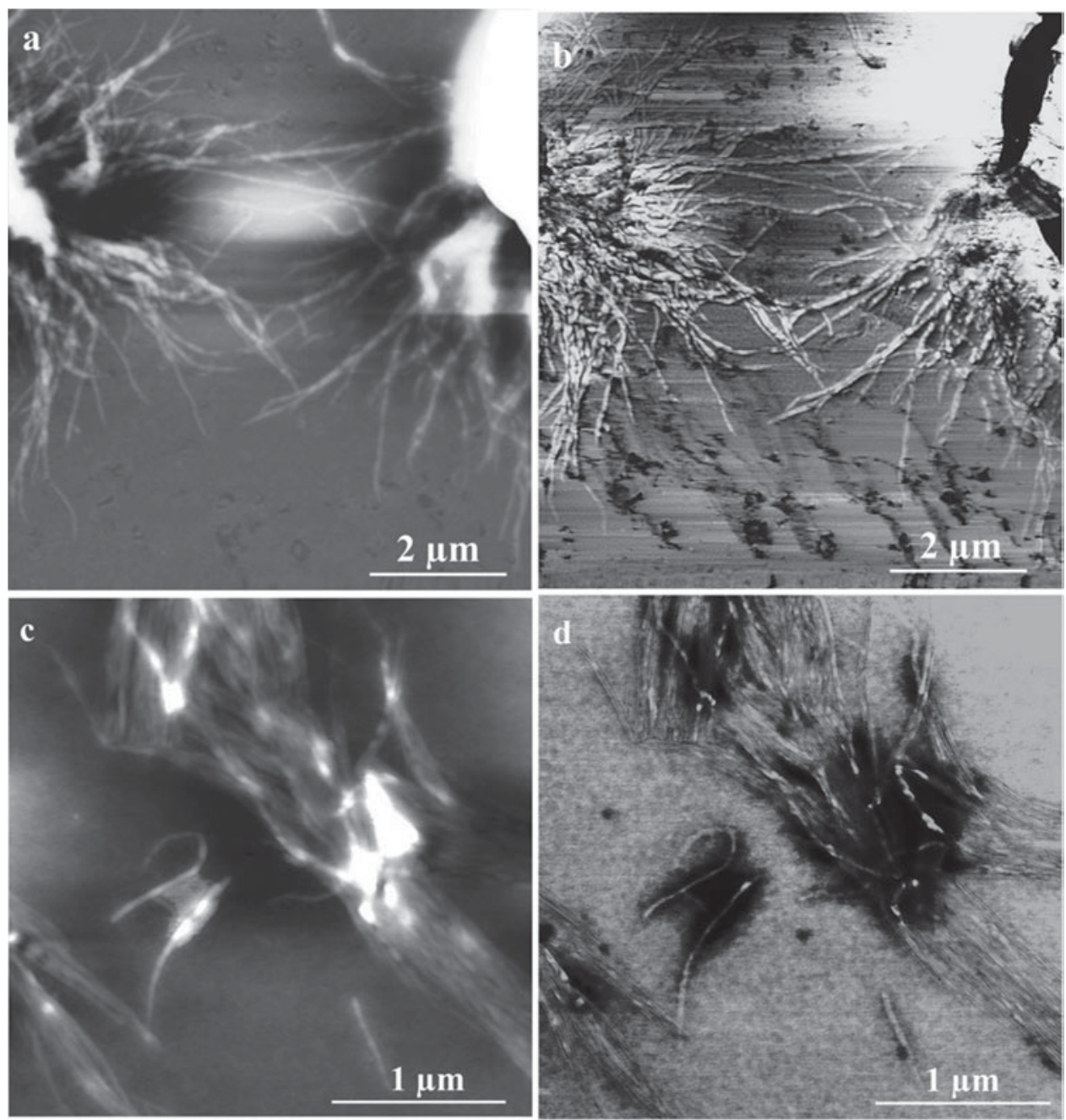

Fig. 2. AFM images of $\alpha$-LaNTs. (a) and (b) shows height and phase images with $R=2$, while (c) and (d) shows those with $R=5$. $R$ : mol $\mathrm{Ca}^{++} / \mathrm{mol} \alpha-$ La.

$\mathrm{Ca}^{++}: \alpha$-la ratio of $2(R=2)$ attached to a glass slide. Figure $2 \mathrm{c}$ and $\mathrm{d}$ show $\alpha$-LaNTs with $R=5$ on a mica surface. Nanotubes seemed to be attached to each other or joined end to end. The length of the linear tubules was as long as $1 \mu \mathrm{m}$ or even longer. Their apparent diameters were in the range of $50-80 \mathrm{~nm}$ (mostly about $70 \mathrm{~nm}$ ). In literature, the apparent width of $\alpha$-LaNTs visualised by SFM (Scanning Force Microscopy) was reported as about $60 \mathrm{~nm}$ (Graveland-Bikker et al. 2006). The difference in the nanotube dimensions, especially in the widths, between AFM and TEM/STEM results arises from a lateral dilation caused by the force microscope tip in AFM with a nominal tip radius in between $48-52 \mathrm{~nm}$.

\section{Raman analyses of $\alpha-l a$, hydrolysates and $\alpha$-LaNTs}

Raman spectroscopy analyses were carried out to investigate the structural changes of $\alpha$-la during hydrolysis and nanotube formation. Figure 3 shows typical Raman spectra of $\alpha$-la protein, its hydrolysates and $\alpha$-la nanotubes. Backbone skeletal region, amide III and amide I regions were presented more closely in Fig. 4. Major peaks detected in each spectra were evaluated based on current literature (Lord \& Yu, 1970; Rava \& Spiro, 1984; Miura et al. 1989; Nonaka et al. 1993; Miura \& Thomas, 1995; Ikeda \& Li-Chan, 2004; Ashton \& Blanch, 2010). They are listed in Supplementary Table 1 accessible in on-line version of the paper (http://www.journals.cambridge.org/dar).

Although many features observed in $\alpha$-la proteins also observed in their hydrolysates and nanotube Raman spectra, there exist clear and important differences between the nanotube and the other Raman spectra. For native $\alpha$-la, amide I region indicated a large loop containing $\alpha$-helix, $\beta$-sheet and disordered structures with the assignments between $1645-1680 \mathrm{~cm}^{-1}$. This broad band is centred at $1657 \mathrm{~cm}^{-1}$ corresponding to a mainly helical structure (Fig. 3). In the hydrolysates, $\beta$-sheets became dominant as evident by the band at $1666 \mathrm{~cm}^{-1}$ (Miura \& Thomas, 1995; Ashton \& Blanch, 2010). The band observed at $1402 \mathrm{~cm}^{-1}$, indicating a symmetric carboxylate stretch, in the hydrolysate spectrum is probably due to the exposure of free 


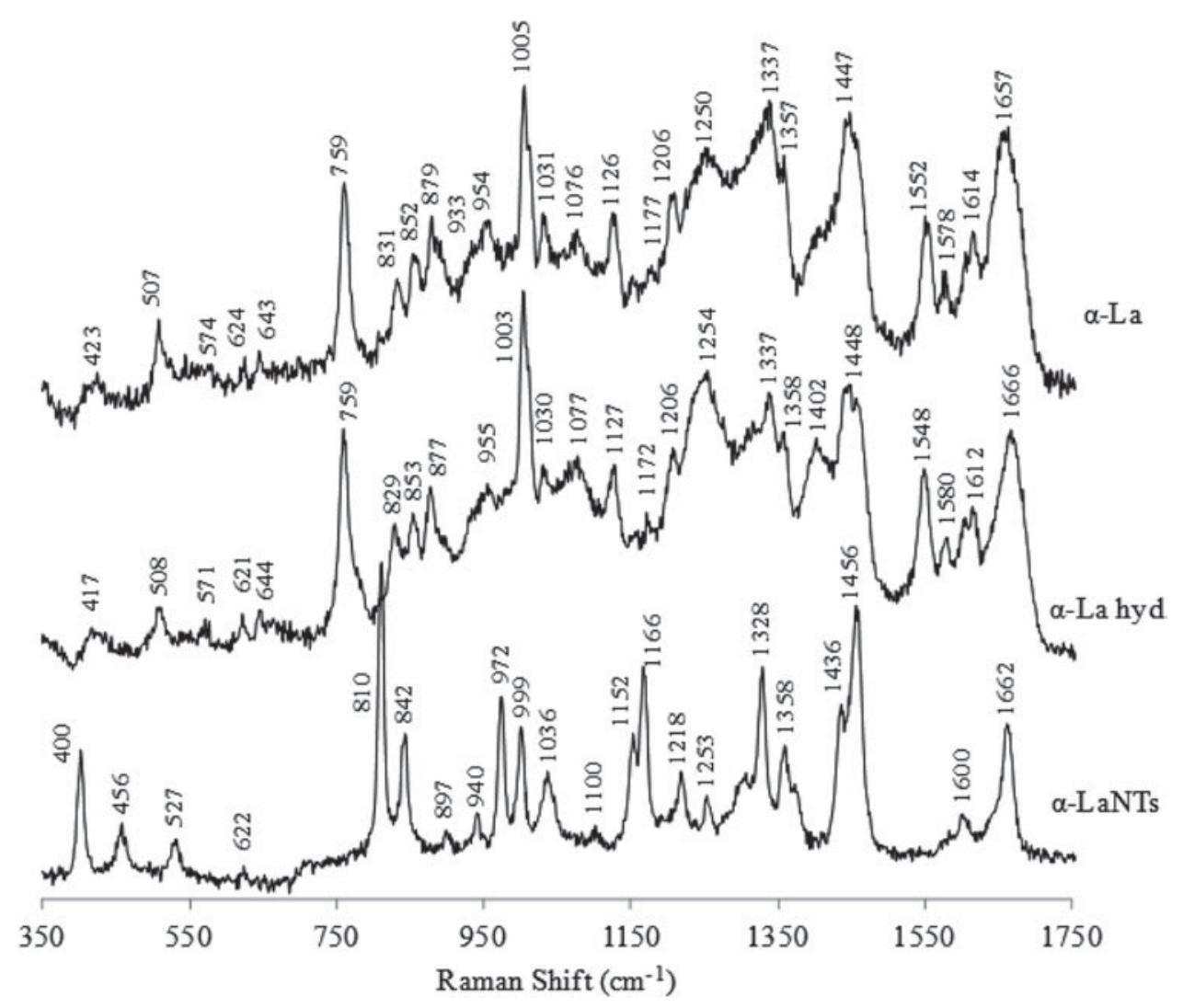

Fig. 3. Raman spectra $\left(250-1750 \mathrm{~cm}^{-1}\right)$ of $\alpha$-La, $\alpha$-La hydrolysates and $\alpha$-LaNTs.
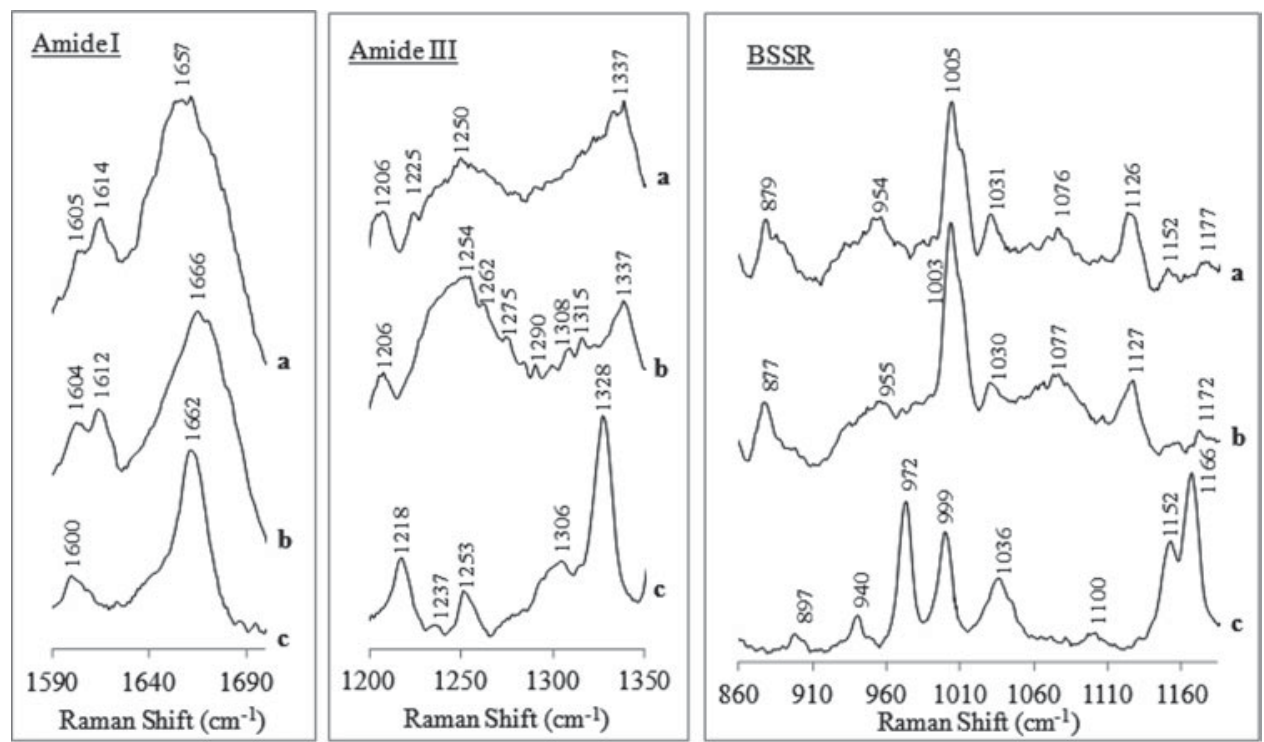

Fig. 4. Smoothed Raman spectra of amide III and BSSR (backbone skeletal stretch region) regions (a) $\alpha$-La, (b) $\alpha$-La hydrolysates and (c) $\alpha$-LaNTs (smoothing achieved by taking average of three neighbouring data).

$\mathrm{COO}^{-}$groups at the digested Asp and Glu sites during the hydrolysis. No peaks at this region were seen in the nanotube spectrum. This is because the peptides are linked by $\mathrm{Ca}^{2+}$ ions at these $\mathrm{COO}^{-}$sites through nanotube elongation. Raman spectrum of the nanotubes exhibited stark differences from that of $\alpha$-la and hydrolysates. Most probably, this is due to a novel conformational arrangement of peptides to form nanotubes. In that case, some bands become Raman active, while some others become Raman inactive. Besides, various shifts and intensity changes are 
also possible. Conformation-sensitive amide I region was seen near $1662 \mathrm{~cm}^{-1}$ with a narrower peak and reduced intensity in the nanotube spectrum. However, the bands at $\sim 1578$ and $1552 \mathrm{~cm}^{-1}$, representing the trptophan side chain in the amide II region had disappeared. Tryptophan band at $\sim 1357 \mathrm{~cm}^{-1}$ gained intensity and sharpness in the nanotube spectrum. The other conformational-sensitive region, amide III, was detected in the region between $\sim 1218$ and $1337 \mathrm{~cm}^{-1}$. Tryptophan band seen at $1337 \mathrm{~cm}^{-1}$ in native $\alpha$-la lost intensity in hydrolysate spectrum. However, in the case of nanotubes two possibilities could be considered. This band might shift to $1328 \mathrm{~cm}^{-1}$ (about $11 \mathrm{~cm}^{-1}$ ) and become sharper, or the peak at $1337 \mathrm{~cm}^{-1}$ become inactive and a new peak at $1328 \mathrm{~cm}^{-1}$ arises from the stretching of new bonds formed during nanotube formation. This band is assigned to $3_{10}$ helix or to residues having local order like that of $3_{10}$ helix at the ends of $\alpha$ - helices or in loops (Wilson et al. 1995; Blanch et al. 2000). The $\alpha$-LaNTs occurs via $\beta$-sheet stacking, leading to 10-start right handed-helix (Graveland-Bikker et al. 2009). The presence of a band around $1310 \mathrm{~cm}^{-1}$ in hydrolysate $\left(1308,1315 \mathrm{~cm}^{-1}\right)$ and nanotube $\left(1306 \mathrm{~cm}^{-1}\right)$ spectrum is attributed to $\alpha$-helix structure (Spiro \& Gaber, 1977). A broad band, assigned as polyproline II-helix, centred at $1250 \mathrm{~cm}^{-1}$ in $\alpha$-la spectrum shifted to $1254 \mathrm{~cm}^{-1}$ in hydrolysate gels with an increased intensity and to $1253 \mathrm{~cm}^{-1}$ in nanotubes with a decreased intensity. Also intensity gains at 1262, 1275, 1290, 1308, and $1315 \mathrm{~cm}^{-1}$ are attributed to the increase in the number of $\alpha$-helix and disordered structures in hydrolysates due to hydrolysis and gel formation (Ikeda \& Li-Chan, 2004). Additionally, the band at $1225 \mathrm{~cm}^{-1}$ detected in protein disappeared in hydrolysates and nanotubes while, a new sharp band appearing at $1218 \mathrm{~cm}^{-1}$ and a weak band at $\sim 1237 \mathrm{~cm}^{-1}$ were detected in nanotubes. The bands at $\sim 1230$ $1245 \mathrm{~cm}^{-1}$ are assigned as $\beta$-sheet structures (Miura \& Thomas, 1995). Also, one at $1218 \mathrm{~cm}^{-1}$ could appear to be a distinct variant of $\beta$-strand or sheet (Barron et al. 2002). Tyrosine and phenylalanine peaks at $\sim 1206 \mathrm{~cm}^{-1}$ had also disappeared in nanotubes. The weak tyrosine and phenylalanine bands at $\sim 1177 \mathrm{~cm}^{-1}$ gained a little intensity in hydrolysates, but in nanotubes, they shifted to $1166 \mathrm{~cm}^{-1}$ with significantly increased intensity or this band disappeared and a new sharp band was born due to stretching of new bonds. Another conformation-sensitive region with backbone skeletal stretchings showed considerable changes especially in the nanotube spectrum. The weak peak at $1152 \mathrm{~cm}^{-1}$, originating mainly from $\mathrm{C}-\mathrm{N}$ stretchings (Nonaka et al. 1993) appeared with an intensity loss in the hydrolysate gels, but with a significant intensity gain in the nanotubes. Tryptophan band at $\sim 1126 \mathrm{~cm}^{-1}$ was also detected in hydrolysates with a small intensity change, but it was not present in the nanotubes. The band attributed to the backbone skeletal stretch at $\sim 1076 \mathrm{~cm}^{-1}$ had gained a little intensity in the hydrolysates and became inactive in the nanotubes also. Phenylalanine peaks at $\sim 1031$ and $1005 \mathrm{~cm}^{-1}$ were expected to shift to 1036 and $999 \mathrm{~cm}^{-1}$ with considerable changes in their intensities. A new peak was detected at $972 \mathrm{~cm}^{-1}$ in the nanotube spectrum. The bands at $\sim 954 \mathrm{~cm}^{-1}$ (assigned as disordered structures), $\sim 879 \mathrm{~cm}^{-1}$ (tryptophan), $\sim 831 / 852 \mathrm{~cm}^{-1}$ (tyrosine Fermi doublet) were also detected in the hydrolysate spectrum with small shifts and intensity changes. However, they had disappeared in the nanotube spectrum which exhibited new bands at 940,897, 842 and $810 \mathrm{~cm}^{-1}$. A distinct Trp band at $759 \mathrm{~cm}^{-1}$ became Raman inactive in the nanotube spectrum. The band around $507 \mathrm{~cm}^{-1}$ is assigned as disulphide bonds in gauche-gauche-gauche conformation, minor bands at around 525 and $540 \mathrm{~cm}^{-1}$ are assigned as those in gauche-gauche-trans and trans-gauche-trans conformations, respectively (Nakanishi et al. 1974; Kitagawa et al. 1979). S-S peak in native $\alpha$-la at $507 \mathrm{~cm}^{-1}$ was also observed in hydrolysates with a decreased intensity and as a broader band, however the minor band at $\sim 542 \mathrm{~cm}^{-1}$ disappeared. Nonaka and co-workers studied the structure of $\alpha$-la gels with Raman spectroscopy and reported that conformation of S-S bonds was altered in the native $\alpha$-la after heating at $90{ }^{\circ} \mathrm{C}$ (Nonaka et al. 1993). Similarly taken data may show the loss of native forms of disulphide bonds by the alteration of conformation due to hydrolysis and gelation. The band representing disulphide bonds was detected at $527 \mathrm{~cm}^{-1}$, instead at $\sim 507 \mathrm{~cm}^{-1}$ in nanotube spectrum, suggesting dominant gauche-gauche-trans conformation, which most probably, arose from a rearrangement of peptides in the nanotubes.

It is reported in the literature (from the CD-spectra analysis) that the secondary structure of the $\alpha$-la is largely retained during nanotube formation (Graveland-Bikker et al. 2009). Our findings also support this idea.

In order to follow the structural changes during the nanotube growth as a function of time, ten-minute samples were taken during incubation and examined with Raman spectroscopy. Figure 5 shows Raman spectra of samples whose incubation times change from 10 to $90 \mathrm{~min}$ with 10 min intervals. It is clear that nanotube spectrum appeared roughly after 60 th min of incubation. Although small shifts and some secondary changes were observed for samples with longer growth durations than $60 \mathrm{~min}$, the assignments were internally consistent. Using the DLS (dynamic light scattering) technique, Graveland-Bikker et al. (2004) found that the nanotube elongation starts at $\sim 50$ th min of incubation. Our findings confirm the literature but using a new approach, that is, Raman spectroscopy. In the Raman spectra of samples up to 50 min incubation, protein and peptide dimmer features were dominant. For samples with longer incubations than this, nanotube spectra were clearly observed. Based on these spectra we can only make qualitative assessments of the observed changes during the nanotube growth as a function of time. We cannot exactly say that the particular nanotube bands increases linearly with time. However, there is a sharp increase in the nanotube band intensities indicating a quick saturation effect in the nanotube concentration. However, Raman results should be evaluated statistically since firstly, each Raman spectrum 


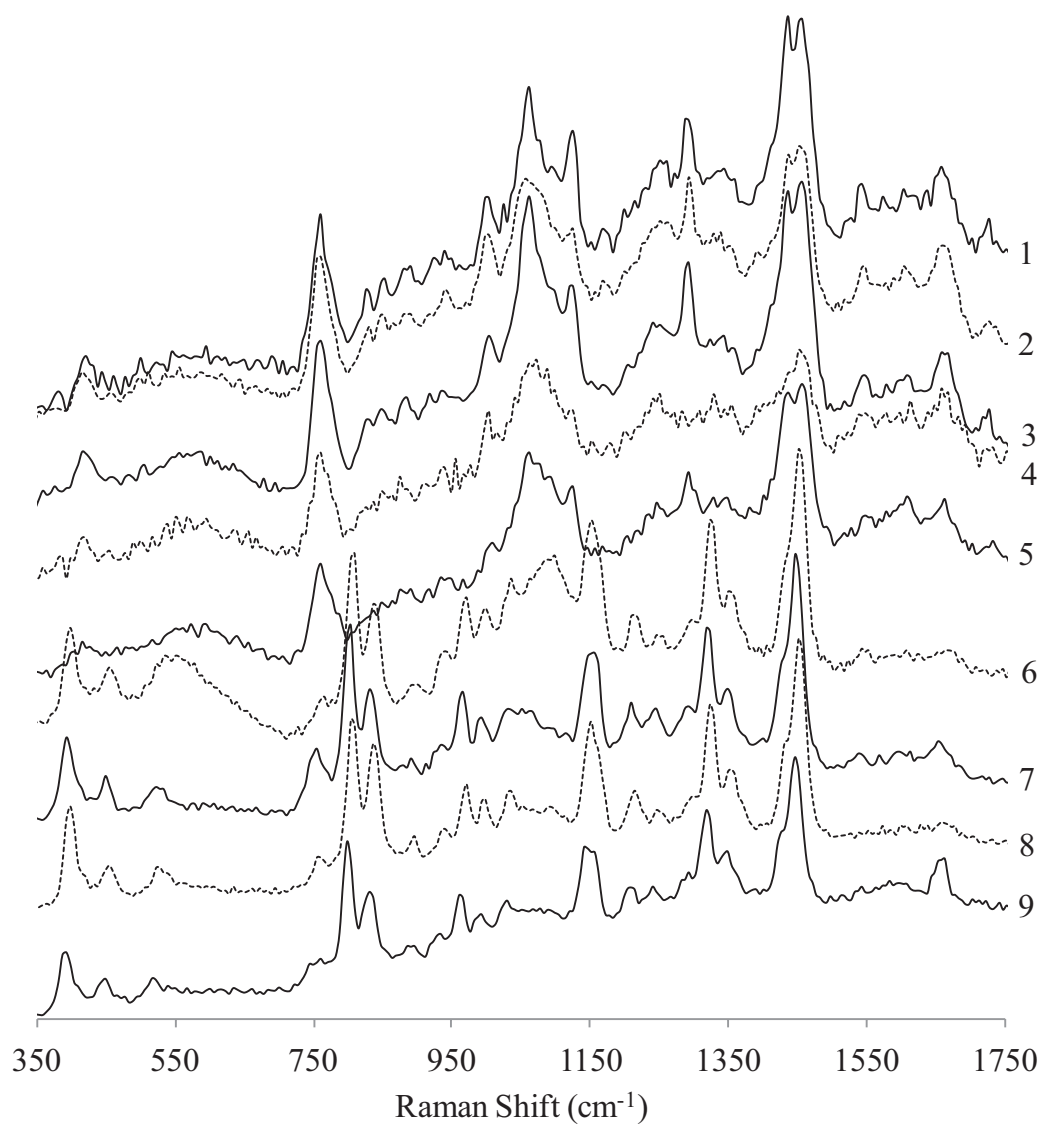

Fig. 5. Raman spectra of the samples taken during nanotube growth with 10 min intervals. Numbers (1-9) indicate sample numbers collected from 10 th to 90 th min along incubation.

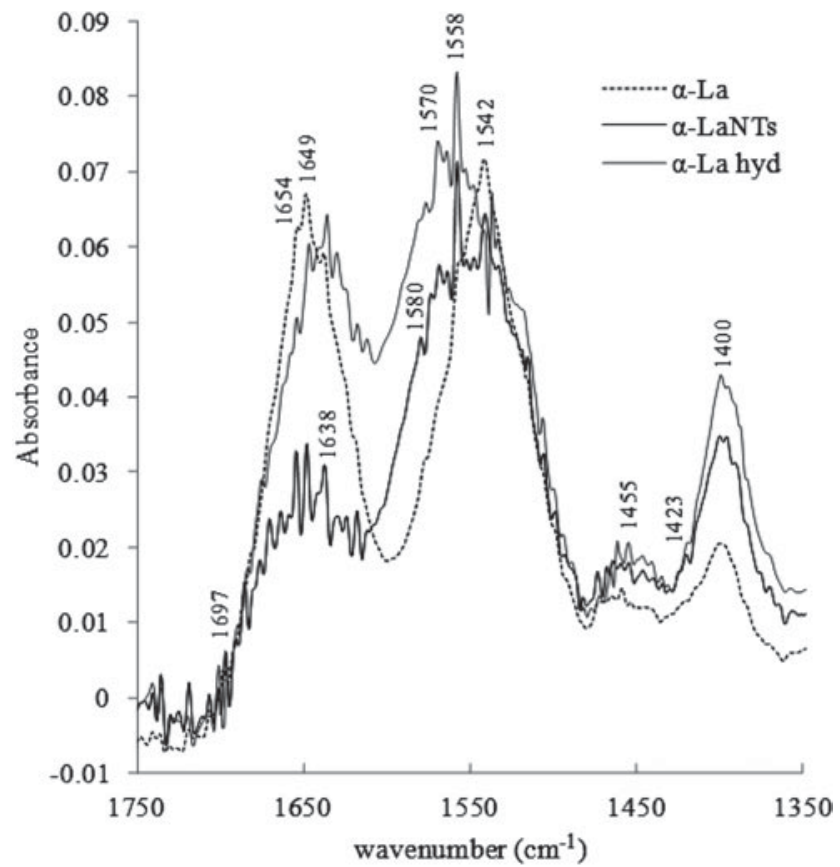

Fig. 6. Fourier-self deconvolved spectra of $\alpha$-La, $\alpha$-La hydrolysates, and $\alpha$-LaNTs. depends strongly on the particular sample point from which the data is taken, and secondly, many samples with same growth durations should be produced for Raman investigations. These tasks require detailed research of its own right. Carrying out such a research for more quantitative results are also planned for our future studies.

\section{FTIR analyses of $\alpha-l a$, hydrolysates and $\alpha$-LaNTs}

We have also carried out FTIR analyses for a better evaluation of structural changes of $\alpha$-la during nanotube formation process and investigation of $\mathrm{Ca}^{++}$binding sites involved in nanotube elongation. Figure 6 represents Fourier-self deconvolved infrared spectra of $\alpha$-la protein, $\alpha$-la hydrolysates and $\alpha$-LaNTs, where the Tris buffer spectrum was subtracted from each. Enlarged amide regions are also given in Fig. 7. Major bands detected in each spectra were analysed by considering current literature (Ambrose \& Elliot, 1951; Anderle \& Mendelsohn, 1987; Jackson \& Mantsch, 1995; Xie \& Liu, 2003; Kong \& Yu, 2007). Supplementary Table 2 listing assignments of major peaks and Supplementary Table 3 listing infrared bands of Asp and Glu side chains are accessible in on-line supplement (http://www.journals.cambridge.org/dar). 

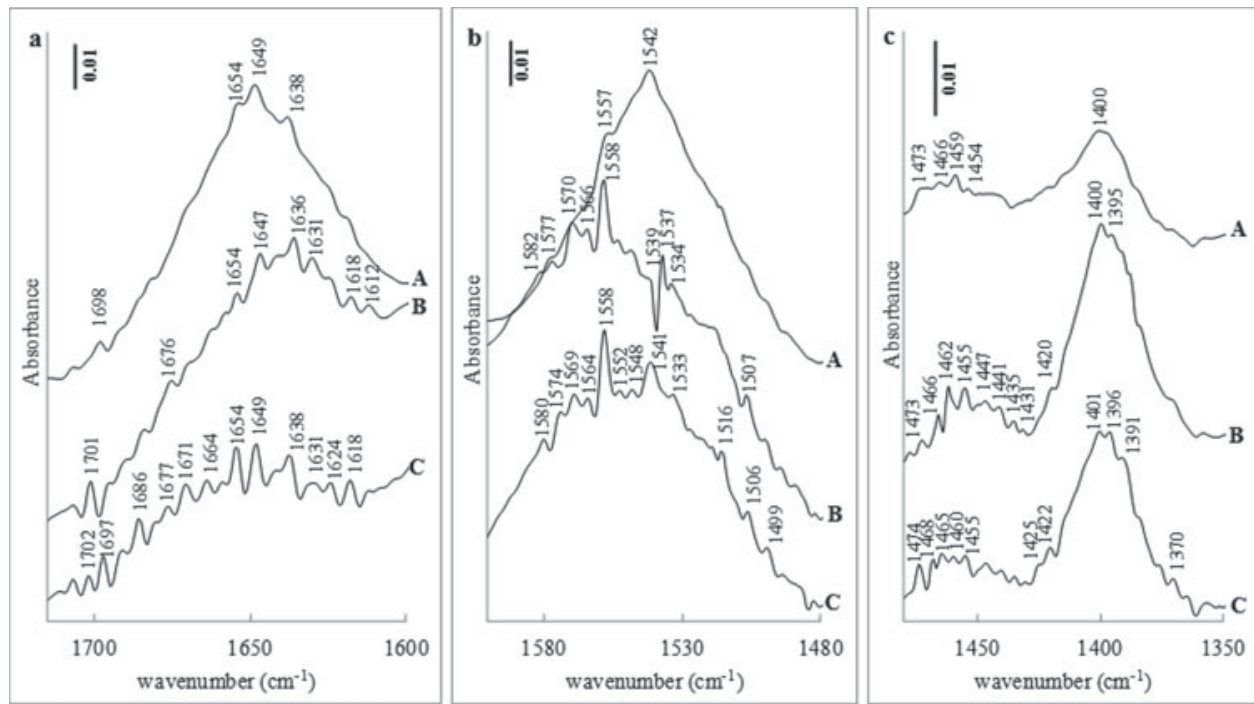

Fig. 7. Fourier-self deconvolved spectra enlarged in the regions of (a) $1715-1600 \mathrm{~cm}^{-1}$; (b) $1600-1480 \mathrm{~cm}^{-1}$; (c) $1480-1350 \mathrm{~cm}^{-1}$. (A) $\alpha$-La, (B) $\alpha$-La hydrolysates and (C) $\alpha$-LaNTs.

In each spectrum, four broad regions are observed. They are assigned as: amide I band at $\sim 1649 \mathrm{~cm}^{-1}$, the $\mathrm{COO}^{-}$ antisymmetric stretching bands at about $1580 / 1558 \mathrm{~cm}^{-1}$ overlapping with amide II band at $\sim 1550 \mathrm{~cm}^{-1}, \mathrm{CH}_{2}$ bending vibration band at $\sim 1460 \mathrm{~cm}^{-1}$, and the $\mathrm{COO}^{-}$ symmetric stretching band at around $1400 \mathrm{~cm}^{-1}$ (Fig. 6). Native $\alpha$-la consists of $\alpha$-helix, $\beta$-sheet and random structures evident by the bands at $1698 / 1638 \mathrm{~cm}^{-1}$ and 1654 / $1649 \mathrm{~cm}^{-1}$. When hydrolysed, the amide I peak intensity decreased slightly, and $\beta$-sheets and turns become dominant which is evident from the new peaks detected between 1612 and $1636 \mathrm{~cm}^{-1}$, and 1676/1701 $\mathrm{cm}^{-1}$ (Fig. 7a). In case of the nanotubes, band intensities decreased significantly and numerous new peaks attributed to all secondary structure elements appeared. They are at around 1618$1638 \mathrm{~cm}^{-1}$ ( $\beta$-sheets/aggregated strands), $1664-1686 \mathrm{~cm}^{-1}$ ( $\beta$-turns), 1697-1702 $\mathrm{cm}^{-1}$ ( $\beta$-sheets). Two possibilities may be considered for this striking intensity loss in the case of the nanotubes: the decrease in the number of bonds giving the corresponding bands displayed in the native protein, and/or the decrease in the activities of these infra-red-active bands of the native protein. After the tertiary structure of $\alpha$-la was disturbed due to the hydrolysis, a new arrangement of peptides for nanotube formation may have caused an increase in the amount of exposed $\beta$-sheets, turns and helix structures.

During hydrolysis of $\alpha-l a$, BLP digests the protein at aspartic (Asp) and glutamic (Glu) acid sites. Carboxy moiety released at Asp and Glu sites available for cation chelation give infrared bands at 1574-1579 and $1404 \mathrm{~cm}^{-1}$ (for Asp), and 1556-1560 and $1402 \mathrm{~cm}^{-1}$ (for Glu) (Barth, 2007). Mizuguchi et al. (1997) examined the relationship between the type of coordination and the stretching of $\mathrm{COO}^{-}$groups in the $\mathrm{Ca}^{++}$binding site of bovine $\alpha$-la. They reported that both $\mathrm{Ca}^{++}$-free and $\mathrm{Ca}^{++}$-binded $\alpha$-la showed two major bands at 1588 and $1578 \mathrm{~cm}^{-1}$ in $\mathrm{COO}^{-}$antisymmetric stretching region with lower intensity in the free form. Also, $\mathrm{Ca}^{++}$binding resulted in the shift at the peak around $1400 \mathrm{~cm}^{-1}$ with splitting two components, 1403 and $1425 \mathrm{~cm}^{-1}$ by gaining intensity, through binding of $\mathrm{Ca}^{++}$ to the $\mathrm{COO}^{-}$groups in the high affinity binding site. Similarly, in our study, the single band at $1400 \mathrm{~cm}^{-1}$ in the native $\alpha$-la appeared as three bands at 1420, 1400 and $1395 \mathrm{~cm}^{-1}$ in hydrolysates by gaining significant intensities (Fig. 7c). In nanotube spectrum, these were detected with a very small shift $\left(\sim 1-2 \mathrm{~cm}^{-1}\right)$ and intensity greater than $\alpha$-la and lower than hydrolysates. Also a shoulder type bands at 1425 and $1391 \mathrm{~cm}^{-1}$ appeared. During hydrolysis, this high affinity calcium binding site should also be exposed, thus giving rise to the intensity increase; however, by the nanotube elongation $\mathrm{Ca}^{++}$ions link the peptides, thus fewer free sites are left which is seen as a decrease in the intensity. Smaller intensity changes were detected in the amide II region, for the three protein samples, compared with amide I region. In the hydrolysates, the band at $1542 \mathrm{~cm}^{-1}$ were lost and two new peaks appeared at 1537 and $1534 \mathrm{~cm}^{-1}$, whereas in the nanotubes the band at $1541 \mathrm{~cm}^{-1}$ retained with lower intensity, and a new band detected at $1533 \mathrm{~cm}^{-1}$ (Fig. 7b). The other $\mathrm{Ca}^{++}$binding site (belonging to of Glu) at $1558 \mathrm{~cm}^{-1}$ gained much intensity in both hydrolysates and nanotubes. Two more signals appeared at 1577 and $1582 \mathrm{~cm}^{-1}$, and 1574 and $1580 \mathrm{~cm}^{-1}$ in the hydrolysate and nanotube spectra, respectively, due to another calcium binding site of aspartic acid. The broad weak band centred at $\sim 1460 \mathrm{~cm}^{-1}$ in protein gained intensity in both hydrolysates and nanotubes. In addition, numerous features were observed between 1431 and $1474 \mathrm{~cm}^{-1}$ arising from $\mathrm{C}-\mathrm{H}$ bending vibrations (Barth, 2007).

In summary, FTIR and Raman Spectroscopy indicated structural conformations in $\alpha$-la during the hydrolysis and nanotube growth. The native tertiary structure of $\alpha$-la was 
destabilised, and some conformational changes were observed in the secondary structure of the protein. The $\beta$-sheets (esp. antiparallel), $\beta$-turns and random structures became dominant due to dimerisation and re-arranged supramolecular structure during nanotube formation. Also, in the helix domain, $3_{10}$ helix structures seemed to gain importance besides $\alpha$-helix. Enzymatic hydrolysis resulted in the exposure of $\mathrm{COO}^{-}$groups at Asp and Glu side chains in the peptides. Then, $\mathrm{Ca}^{++}$ions were predicted to bind peptides at these groups which led mainly to the nanotube elongation. FTIR spectroscopic analysis showed some shifts, gain and lose of intensities at the related bands due to $\mathrm{Ca}^{++}$ binding to $\mathrm{COO}^{-}$groups of these specific sites.

\section{Supplementary material}

The supplementary material for this article can be found at http:// www.journals.cambridge.org/dar

We would like to thank Associate Professor Banu Özen for her help in the FTIR analyses. We also thank to Central Lab in METU for TEM analyses and Center for Material Research in IZTEC for STEM and AFM analyses. This work was supported financially by the The Scientific and Technological Research Council of Turkey (109O866, 2010-2011) and izmir Institute of Technology, Scientific Research Projects fund (IYTE03, 2009-2011).

\section{References}

Ambrose EJ \& Elliot A 1951 Infrared spectroscopic studies of globular protein structure. Proceedings of the Royal Society London Series A 208 75-90

Anderle G \& Mendelsohn R 1987 Thermal denaturation of globular proteins Fourier transform-infrared studies of the amide III spectral region. Biophysical Journal 52 69-74

Ashton L \& Blanch EW 2010 pH-induced conformational transitions in $\alpha$-lactalbumin investigated with two-dimensional Raman correlation variance plots and moving windows. Journal of Molecular Structure 947 132-138

Barron LD, Blanch EW \& Hecht L 2002 Unfolded proteins studied by Raman optical activity. Advanced in Protein Chemistry 62 51-90

Barth A 2007 Infrared spectroscopy of proteins. Biochimica et Biophysica Acta 1767 1073-1101

Blanch EW, Morozova-Roche LA, Hecht L, Noppe W \& Barron LD 2000 Raman optical activity characterization of native and molten globule states of equine lysozyme: comparison with hen lysozyme and bovine $\alpha$-lactalbumin. Biopolymers (Biospectroscopy) 57 235-248

Breddam K \& Meldal M 1992 Substrate preferences of glutamic-acid-specific endopeptidases assessed by synthetic peptide substrates based on intramolecular fluorescence quenching. European Journal of Blochemistry 206 103-107

Graveland-Bikker JF \& de Kruif CG 2006 Unique milk protein based nanotubes: food and nanotechnology meet. Trends in Food Science and Technology 17 196-203

Graveland-Bikker JF, Ipsen R, Otte J \& de Kruif CG 2004 Influence of calcium on the self-assembly of partially hydrolyzed $\alpha$-lactalbumin. Langmuir 20 6841-6846

Graveland-Bikker JF, Schaap IAT, Schmidt CF \& de Kruif CG 2006 Structural and mechanical study of a self-assembling protein nanotube. Nano Letters 6 616-621

Graveland-Bikker JF, Koning RI, Koerten HK, Geels RBJ, Heeren RMA \& de Kruif CG 2009 Structural characterization of $\alpha$-lactalbumin nanotubes. Soft Matter 5 2020-2026
Ikeda S \& Li-Chan ECY 2004 Raman spectroscopy of heat-induced finestranded and particulate $\beta$-lactoglobulin gels. Food Hydrocolloids $\mathbf{1 8}$ 489-498

Ipsen R \& Otte J 2003 Nano-structuring by means of proteolysis rheology of novel gels from $\alpha$-lactalbumin. Annual Transactions of the Nordic Rheology Society 11 89-93

Ipsen R \& Otte J 2007 Self-assembly of partially hydrolysed $\alpha$-lactalbumin. Biotechnology Advances 25 602-605

Ipsen R, Otte J \& Qvist KB 2001 Molecular self-assembly of partially hydrolysed $\alpha$-lactalbumin resulting in strong gels with a novel microstructure. Journal of Dairy Research 68 277-286

Ipsen R, Otte J \& Qvist KB 2003 Protease induced nano-tubular gels from $\alpha$-lactalbumin. In Food Colloids, Biopolymers and Materials, Special Publication, Vol. 284, pp. 84-92 (Eds E Dickinson \& T van Vliet). London: Royal Society of Chemistry

Jackson M \& Mantsch HH 1995 The use and misuse of FTIR spectroscopy in the determination of protein structure. Critical Reviews in Biochemistry and Molecular Biology 30(2) 95-120

Kitagawa T, Azuma T \& Hamaguchi K 1979 The Raman spectra of Bence-Jones proteins. Disulfide stretching frequencies and dependence of Raman intensity of tryptophan residua on their environments. Biopolymers 18 451-465

Kong J \& Yu S 2007 Fourier Transform Infrared Spectroscopic Analysis of protein secondary structures. Acta Biochimica et Biophysica Sinica 39 $549-559$

Krimm S \& Bandekar J 1986 Vibrational spectroscopy and conformation of peptides, polypeptides, and proteins. Advanced in Protein Chemistry $\mathbf{3 8}$ $181-367$

Lord RC \& Yu NT 1970 Laser-excited Raman spectroscopy of biomolecules. I. Native lysozyme and its constituent amino acids. Journal of Molecular Biology 50 509-524

Miura T \& Thomas GJ Jr 1995 Raman spectroscopy of proteins and their assemblies. In Subcellular Biochemistry (Proteins: Structure, Function and Engineering), Vol. 24, pp. 55-99 (Eds BB Biswas \& S Roy) New York: Plenum

Miura T, Takeuchi H \& Harada I 1989 Tryptophan Raman bands sensitive to hydrogen bonding and side-chain conformation. Journal of Raman Spectroscopy 20 667-671

Mizuguchi M, Nara M, Kawano K \& Nitta K 1997 FT-IR study of the $\mathrm{Ca} 2+$-binding to bovine $\alpha$-lactalbumin. FEBS Letters 417 153-156

Nakanishi M, Takesada H \& Tsuboi M 1974 Conformation of the cystine linkages in bovine $\alpha$-lactalbumin as revealed by its Raman effect. Journal of Molecular Biology 89 241-243

Nara M, Tasumi M, Tanokurab M, Hiraoki T, Yazawa M \& Tsutsumi A 1994 Infrared studies of interaction between metal ions and $\mathrm{Ca}^{\prime}$-binding proteins. FEBS Letters 349 84-88

Nonaka M, Li-Chan E \& Naki S 1993 Raman spectroscopic study of thermally induced gelation of whey proteins. Journal of Agricultural and Food Chemical 41 1176-1181

Rajagapol K \& Schneider JP 2004 Self-assembling peptides and proteins for nanotechnological applications. Current Opinion in Structural Biology 14 480-486

Rava RP \& Spiro TG 1984 Ultraviolet resonance Raman spectra of insulin and $\alpha$-lactalbumin with 218-and 200-nm laser excitation. Biochemistry 24 1861-1865

Spiro TG \& Gaber BP 1977 Laser Raman scattering as a probe of protein structure. Annual Review of Biochemistry 46 553-572

Tu AT 1986 Peptide backbone conformation and microenvironment of protein side chains in spectroscopy of biological systems In: Spectroscopy of Biological Systems, pp. 47-112 (Eds Clark RJH \& Haster RE) New York: John Wiley \& Sons

Wilson G, Ford SJ, Cooper A, Hecht L, Wen ZQ \& Barron LD 1995 Vibrational Raman optical activity of alpha-lactalbumin: comparison with lysozyme, and evidence for native tertiary folds in molten globule states. Journal of Molecular Biology 254 747-760

Xie M-X \& Liu Y 2003 Studies on amide III infrared bands for the secondary structure determination of proteins. Chemical Journal of Chinese Universities 24 226-231 